\title{
La hidrogeología del entorno del Parque Nacional de la Caldera de Taburiente, La Palma (Islas Canarias)
}

\author{
Annetty Benavides Gelabert \\ Ilustre Colegio Oficial de Geólogos. Colegiada N 7468. \\ Ataúlfo Argenta, 4 -1H3. Las Palmas de Gran Canaria. 35013 \\ annettybg@gmail.com
}

\begin{abstract}
RESUMEN
La hidrogeología del entorno del Parque Nacional de la Caldera de Taburiente está condicionada por un contexto geológico volcánico caracterizado por la presencia de varios edificios subaéreos, un basamento impermeable de origen submarino, y grandes discordancias erosivas que influyen en la circulación de las aguas subterráneas. En la primera parte de este trabajo se sintetizan aspectos considerados esenciales de la geología, hidroquímica e isotopía ambiental de la zona, a partir del conocimiento generado en varios estudios realizados en las últimas décadas. En segundo lugar, se presenta una estimación del balance hidrometeorológico del acuífero central (Coebra) -que alimenta a manantiales y galerías tanto en el interior como exterior de la Caldera- calculado a partir de datos meteorológicos disponibles para el período 1985-2015.
\end{abstract}

Palabras clave: Caldera volcánica, hidroquímica, isótopos, balance hidrometeorológico.

\section{Hydrogeology of the Caldera de Taburiente National Park area, La Palma (Canary Islands)}

\begin{abstract}
The hydrogeology of the Caldera de Taburiente National Park area is influenced by a geological scenario characterized by several sub-aerial volcanic edifices lying on an impermeable basement of submarine volcanic origin, and the presence of extensive erosive unconformities defining the groundwater flow. The first part of this paper presents a summary of the essential aspects on the geology, hydrogeochemistry and isotopic chemistry of the area, based on the knowledge generated by studies carried out in the last decades. The second part provides an approach to the hydro-meteorological balance of the Coebra central aquifer, which feeds springs and galleries both inside and outside the Caldera, calculated from available meteorological data for the period 1985-2015.
\end{abstract}

Keywords: volcanic caldera, hydrochemistry, isotopes, water balance.

\section{ABRIDGED ENGLISH VERSION}

\section{Introduction}

The Caldera de Taburiente, on the island of La Palma (Fig. 1) has long been highly appreciated due to springs emerging at its margins. Hydrogeologically, the area is characterized by the existence of an aquifer located on the highest and most central part of the north shield. The aquifer discharges through springs located geomorphologically at higher levels, both in the inner rim of the Caldera and the outer edge (Marcos and Cordero springs). At the same time, the aquifer is exploited through water galleries (Fig. 2). Bravo and Coello (1979) described a great erosive unconformity in the form of a bucket, forming the aquifer that gives rise to the springs and galleries inside the Caldera de Taburiente. This structure was later called the "Coebra" in the Insular Hydrological Plan (Navarro, 1992) after the researchers Juan Coello and Telesforo Bravo (Fig. 3). In the 
Annetty Benavides Gelabert, 2019. La hidrogeología del entorno del Parque Nacional de... Boletín Geológico y Minero, 130 (4): $641-660$

Geological Map of Spain (Carracedo et al., 2015) (Fig. 4) this unconformity giving rise to the Coebra structure, is attributed to a gravitational megalandslide in the Garafía volcano (1.2 Ma) and its further filling by the eruptive activity after the collapse (Lower Taburiente volcanic edifice) forming the so-called aquifer of the central plateau of horizontal lavas.

\section{Hydrochemical and isotopical characterisation}

Several studies carried out in the area (COP, 1987; Veeger, 1991; König, 1997; Poncela, 2015) have reported spatial variations in the quality of the water. Groundwater sampled in springs and galleries located at high altitudes, both inside and outside the Caldera, show less mineralization and have been related to the central aquifer(Coebra) (Fig.5, Tab.1). The hydrochemical classification based on the dominant ions results in the majority of the waters being classified as bicarbonated, and with a more variable cationic content (Fig. 6). However, groundwater sampled in springs and galleries of the lower zone of the Caldera shows higher values of temperature and mineralisation, and a more sulphated magnesium calcium composition trend than those related to the Coebra aquifer.

The study of the stable isotopes of the La Palma water has been addressed in several studies carried out on the island (Veeger, 1991; ITGE, 1993; König, 1997; Poncela, 2015). The resulting local meteoric lines differ from each other, although the sampling conditions of the selected rainfall and springs and the date of completion are also different in each case. Figure 7 shows that the most isotopically enriched samples correspond to the springs of the lower zone of the Caldera, whilst the lighter ones are related to the Coebra aquifer (Fig.7). The relationship between the isotopic content and elevation also shows a delimitation of the aquifers according to the recharge altitude (Fig. 8). The samples located at the base of the Caldera reflect variable recharge levels, but in general they do not exceed 1,400 m, much lower than those linked to the Coebra aquifer, related to the highest recharge elevation of between 1,400 and 2,000 m.

Considering the model proposed by PONCELA (2015) and based on recent Tritio determinations, we propose that the groundwater in the highest elevations of the northern cone, coinciding with the Coebra sector and its outer slopes, comes from recent recharge waters.

\section{Water Balance}

An approximation to the hydrometeorological balance has been made from the few available monthly meteorological data sets in the area (Fig. 9). Gap filling has been addressed through correlational analysis applying the methodology used by Heredia and Meléndez (2016) in the sub-basin of the Pas River in Puente Viesgo (Cantabria). Tables 2 and 3 indicate the sequential process of the filling. According to the purposes of this study, the limits of the system have been determined from surface geology (Carracedo et al, 2001).

Zonal calculation of rainfalls $(\mathrm{mm})$ is carried out by the Thiessen polygons method (Fig. 10). The average annual precipitation value obtained for the Coebra area is $917 \mathrm{~mm}$. Different climatic scenarios have also been evaluated based on a deviation of $20 \%$ with respect to the average, resulting in average values of 580 $\mathrm{mm}$ and $1,372 \mathrm{~mm}$ for dry and wet years respectively.

Zonal evapotranspiration calculation is more complex given the few thermometric stations existing in the area. For this reason, and considering the close relationship between temperature and altitude in the islands of the archipelago, some virtual stations have been introduced. The relationship between altitude and temperature (Fig. 11) is evidenced by high coefficients of determination $\left(R^{2}\right)$ resulting from the correlational analysis carried out with these two variables by using the previously filled series (Fig. 12). The calculation of the evapotranspitation has been addressed by the classical method of Thornthwaite (1948) and by the simplified formula of Hargreaves (Hargreaves \& Samani, 1985). Table 4 presents the result of the hydrometeorological balance for the period 1985-2015 from values of PET / ET $T_{0}$ calculated by Tornthwaite and Hargreaves, respectively. In the absence of soil parameters, the available water content of the soil was considered uniformly 50 $\mathrm{mm}$ and $10 \mathrm{~mm}$, whilst the estimate of direct runoff has been addressed by the empirical method of the National Resource Conservation Service of the United States (NRCS, 2004).

\section{Conclusions}

From several hydrogeological studies carried out around the Caldera de Taburiente National Park, two areas of circulation and quality of groundwater can be delimited. The springs and galleries located at high altitudes are related to the Coebra aquifer, formed by an erosive unconformity associated with a large landslide. The waters of this aquifer have sodium bicarbonate composition and very low mineralization, whilst the ground- 
water of the lower zone of the Caldera, associated with the Pliocene materials, presents a lower flow rate and greater mineralization.

The analysis of stable isotopes also reflects zonal groundwater delimitation. Higher recharge levels are associated to Coebra aquifer, showing a greater relationship with the local rainfall according to the slope orientation, and a vertical permeability predominance favoured by the numerous dyke intrusions. However, the underground flow inside and outside its limits, as well as the underground extension of the aquifer and hydraulic functioning of the system still bring up many open questions.

The estimation of the water balance of the Coebra sector is uncertain given the lack of available data, the use of statistical methods as well as the monthly scale used. Moreover, the results are different depending on the calculation method of the potential evapotranspiration PET/ET (Thornthwaite/Hargreaves). Nevertheless, the results provide a first approximation to the natural recharge value of the aquifer with the aim of calculating the water balance of the area.

\section{Introducción}

La Caldera de Taburiente, en la isla de La Palma (Fig. 1), ha sido apreciada desde antaño debido a las aguas de los manantiales (en Canarias, nacientes) que afloran en sus márgenes. Numerosos documentos desde el Siglo XV describen como río o arroyo abundante, al flujo continuo de agua que se forma de la unión de varias fuentes que surgen del interior de sus laderas, y que se unen fluyendo por el Barranco de las Angustias. Las aguas de estos manantiales, y posteriormente de las galerías ejecutadas en el pasado siglo, han sido históricamente aprovechadas para la agricultura.

En general, el funcionamiento del acuífero insular se corresponde con el modelo conceptual de los acuíferos insulares volcánicos (SPA-15, 1975; Custodio and Cabrera, 2008) en el que se define un único acuí- fero heterogéneo y anisótropo, donde la recarga se produce en las zonas altas y de medianías, y fluye hacia la costa, con diferencias locales según los condicionantes geológicos. En particular, la zona de estudio se caracteriza por el emplazamiento de un acuífero central (Coebra) a las mayores altitudes del escudo norte insular, el cual realiza parte de su descarga a través de manantiales situados a cotas elevadas (>1000 m s. n. m.), tanto en el interior como en el exterior de la Caldera (nacientes Marcos y Cordero), a la vez que es explotado por varias galerías que lo penetran (Fig. 2). En cambio, en la zona baja de la Caldera, la circulación de aguas subterráneas es más reducida y los manantiales son de poco caudal, siendo muy cuantiosa la escorrentía superficial durante períodos lluviosos.

A continuación se presentan los aspectos considerados más relevantes del conocimiento generado en

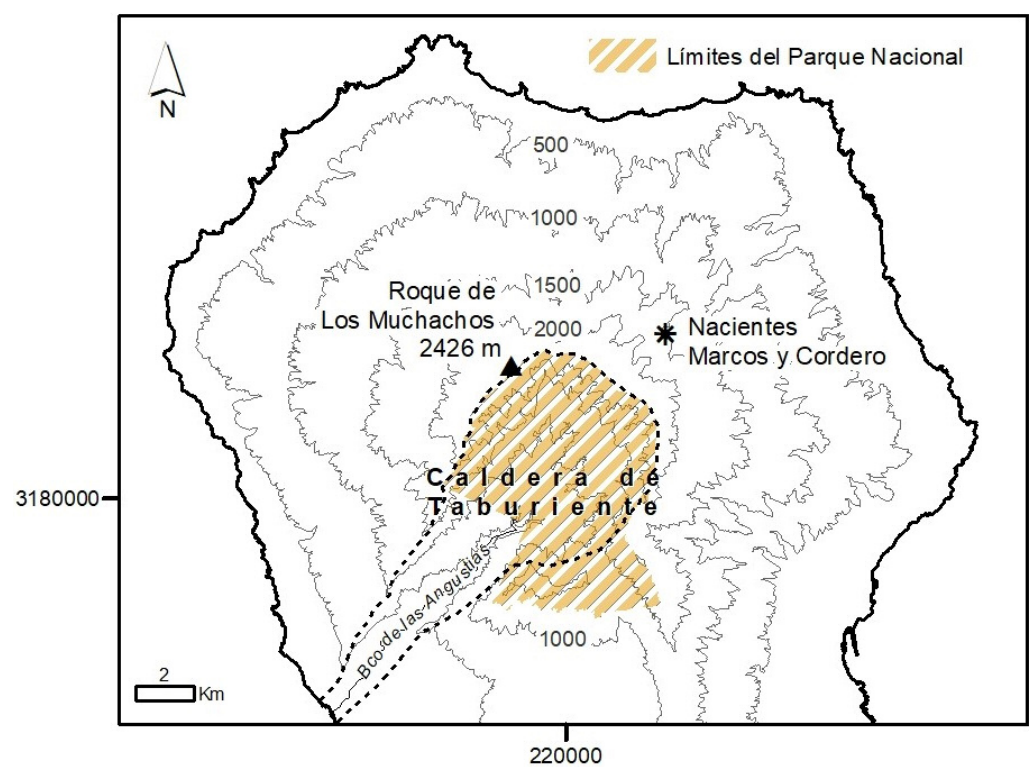

\section{Islas Canarias}

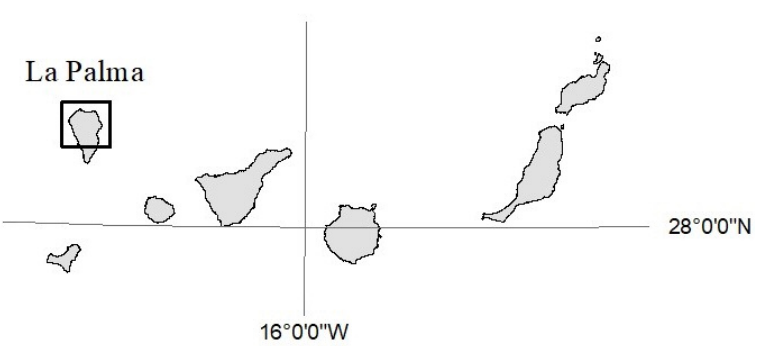

Figura 1. Localización del Parque Nacional de La Caldera de Taburiente. Figure 1. Location of the Caldera de Taburiente National Park. 
torno a la hidrogeología de la Caldera de Taburiente, con énfasis en el acuífero central o Coebra, considerado un reservorio de agua de gran calidad, fuente de alimentación de manantiales y galerías que existen dentro del área del Parque Nacional de la Caldera de Taburiente, y de cuya existencia depende el valioso ecosistema que contiene.

\section{Encuadre geológico}

Desde el siglo XIX se han realizado numerosos estudios sobre la geología de La Palma, y en especial sobre la Caldera de Taburiente. Destacan los de Leopold von Buch quien interpreta su origen geológico mediante su teoría de los cráteres de elevación (von Buch, 1825) y emplea por primera vez el término caldera, ya usado por los habitantes de Canarias para dar nombre a esta enorme depresión rodeada de abruptas paredes. Posteriormente, Charles Lyell, en la edición ampliada de su Elements of Geology (Lyell,
1865), analiza críticamente este origen geológico atribuyéndole un carácter erosivo, e incorpora de una manera más amplia el término caldera a la literatura geológica.

En etapas posteriores, se realizan diversos estudios que van incorporando conocimientos sobre la geología de la Caldera de Taburiente y el resto de la isla. En el mapa geológico del proyecto SPA-15 (1975), de gran trascendencia para la hidrogeología de Canarias (Cabrera and Custodio, 2011), se distinguen en el Norte de la isla dos formaciones principales desde el punto de vista hidrogeológico. En primer lugar el Complejo Basal, aflorante en el fondo de la Caldera, formado por materiales volcánicos submarinos e intrusiones magmáticas, y que constituye el núcleo impermeable. Recubriendo éste, sobre una base formada por aglomerados, se asienta un apilamiento de coladas basálticas que alcanza los mil metros de espesor, distinguiéndose varias series, a través de las cuales se produce el flujo de las aguas subterráneas desde las mayores cotas hacia la costa.
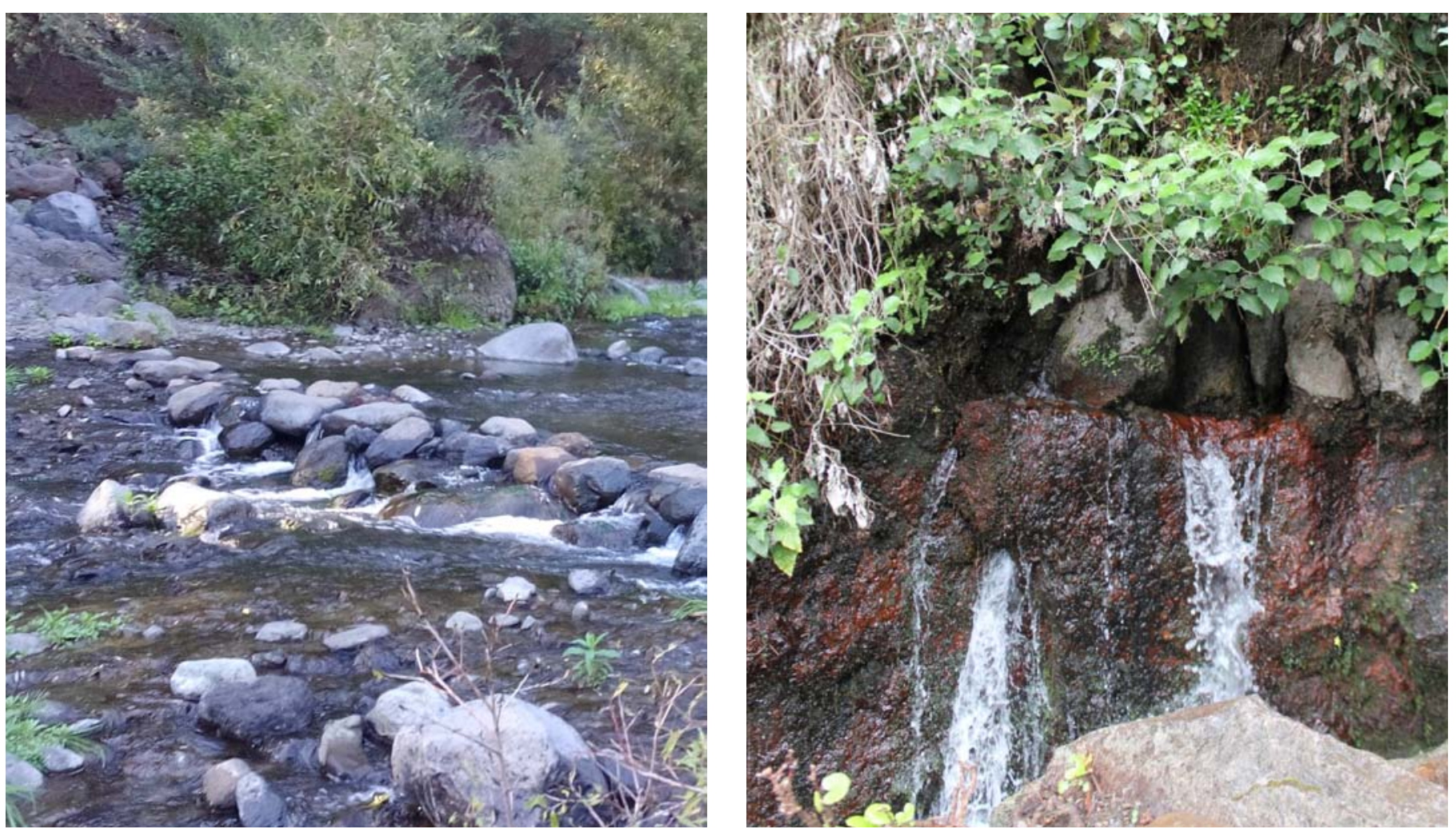

Figura 2. A.) Las aguas procedentes de galerías y manantiales del interior de la Caldera, a las que se incorpora la escorrentía superficial durante episodios lluviosos, circulan por el cauce hasta el punto de distribución para su uso en la agricultura. B). El naciente Cordero brota en el contacto discordante entre las lavas horizontales del edificio volcánico Taburiente Inferior y los materiales del edificio volcánico Garafía.

Figure 2. A) Waters from galleries and springs located inside the Caldera, circulate along the riverbed towards the distribution site for agricultural purposes. Surface runoff waters are joined to the flow during rainy seasons. B). The Cordero spring flows emerging along an unconformable surface between horizontal lavas of LowerTaburiente volcano and the materials of Garafía volcano. 
Bravo y Coello (1979) y Coello (1987) realizan aportaciones importantes, basadas en las perforaciones de galerías ejecutadas en el norte de la Palma. En estos trabajos se describe la existencia de una gran discordancia erosiva en las series subaéreas, atribuida a paleocalderas que habrían sido rellenadas posteriormente por coladas de lavas en posición horizontal. Esta estructura en forma de cubeta, constituye el acuífero que da lugar a los manantiales y galerías en el interior de la Caldera de Taburiente, Ilamado posteriormente "Coebra" en el Plan Hidrológico Insular en honor a los investigadores Juan Coello y Telesforo Bravo. (Fig. 3).

En el Mapa Geológico de España (Carracedo et al., 2015) las unidades volcanoestratigráficas se separan en edificios volcánicos: el Submarino, coincidente esencialmente con el Complejo Basal, y el Subaéreo, separado a su vez en otros edificios volcánicos definidos según geocronología (Fig. 4). En dicho trabajo se revisa la interpretación exclusivamente erosiva de la Caldera de Taburiente, proponiendo su origen debido a un gran deslizamiento gravitacional (Cumbre Nueva, $560 \mathrm{Ka}$ ) y su posterior erosión. Así mismo, la discordancia sobre la que se asientan las lavas horizontales que constituyen la estructura Coebra se atribuye a otro megadeslizamiento gravitacional en el edificio volcánico Garafía (1,2 Ma) y su relleno por la actividad eruptiva posterior al colapso (edificio volcánico Taburiente Inferior), formando el denominado acuífero de la meseta central de lavas horizontales (Coebra).
La recarga de este acuífero depende de la infiltración directa de las aguas de lluvia que caen en su vertical, presentando una alta permeabilidad en superficie debido a una geología formada por secuencias de lavas basálticas y niveles escoriáceos del edificio volcánico Taburiente. Debido a la presencia de materiales aglomeráticos, el plano del deslizamiento Garafía se comporta como un límite impermeable que retiene parcialmente el flujo radial regional del agua subterránea. La zona saturada se localiza en los materiales suprayacentes, las lavas horizontales del edificio Taburiente Inferior, donde la abundante presencia de diques produce la compartimentación y sobreelevación local de los niveles piezométricos, con predominio de la permeabilidad vertical. El afloramiento de esta discordancia geológica da lugar a importantes surgencias, tanto en el borde interior como en el exterior de la Caldera (nacientes Marcos y Cordero). A su vez, varias galerías penetran el reservorio para su explotación, si bien la mayoría presentan cierres hidráulicos a través de los cuales se regula convenientemente los caudales.

En la zona deprimida de la Caldera afloran los materiales pliocenos del edificio submarino, predominando las lavas almohadilladas, brechas, aglomerados y hialoclastitas altamente metasomatizados, así como intrusiones de gabros. La antigüedad y alteración del conjunto confieren a esa zona muy baja permeabilidad, favoreciendo una alta y rápida escorrentía superficial durante episodios lluviosos.

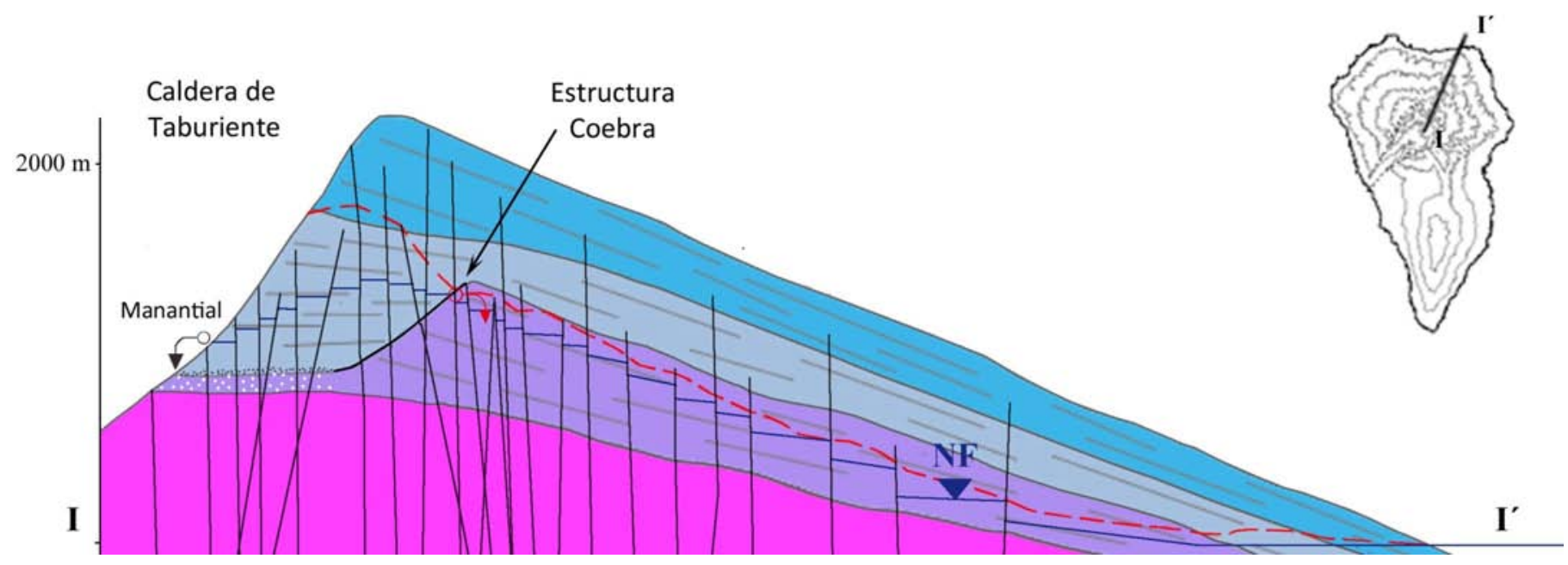

Figura 3. Corte esquemático de la estructura Coebra (modificado de Navarro, 1992). La línea discontinua roja representa el caso del Barranco del Agua, donde la erosión ha dejado al descubierto la estructura Coebra (cabecera del deslizamiento Garafía) dando lugar a los nacientes Marcos y Cordero (para la simbología véase Fig. 4).

Figure 3. Schematic cross-section of Coebra structure (modified from Navarro, 1992). The red dashed line represents the case of the Barranco del Agua, where erosion has exposed the Coebra structure (Garafía head of landslide) giving rise to the Marcos and Cordero springs (see Fig. 4 for legend). 

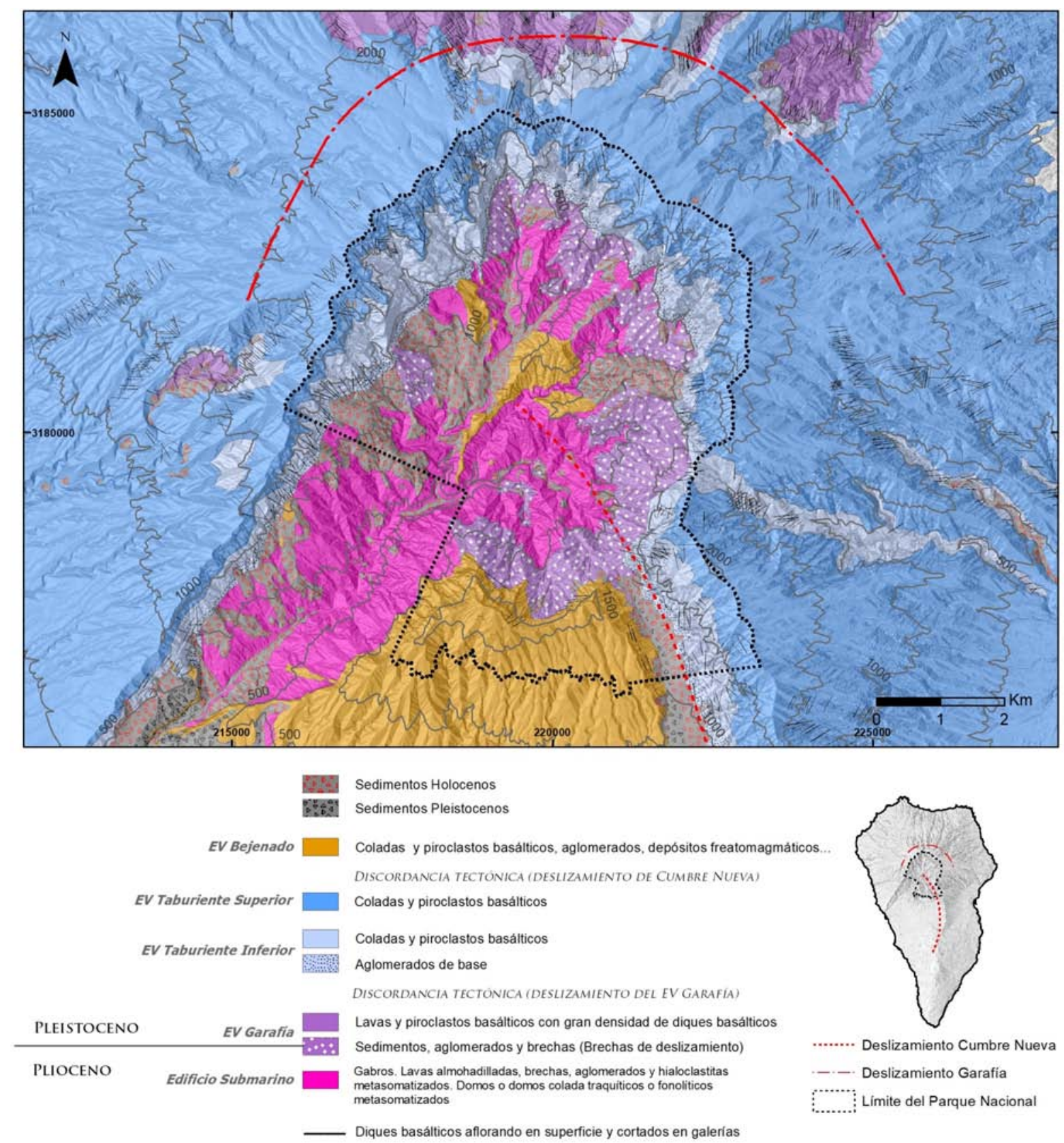

Figura 4. Mapa geológico del área del Parque Nacional de la Caldera de Taburiente, simplificado del GEODE (Bellido et al, 2004) y Carracedo (2015).

Figure 4. Geological map of the Caldera de Taburiente National Park area, simplified from GEODE (Bellido et al, 2004) and Carracedo (2015).

\section{Caracterización hidrogeoquímica}

La baja mineralización de las aguas subterráneas del norte de La Palma ha quedado manifiesta desde la realización del proyecto SPA-15 (1975), lo que se relaciona a su mayor pluviometría, muy condicionada por la orientación de las vertientes, y al rápido flujo subterráneo, favorecido por la alta permeabilidad ver- tical por la presencia de diques. Por lo general, en los manantiales y galerías de la zona norte predominan las aguas bicarbonatadas sódicas o bicarbonatadas mixtas presentando un elevado $\mathrm{pH}(>8)$, habitual en terrenos basálticos dada su facilidad alteración, en especial del olivino (Custodio and Llamas, 1987).

En particular, el área de la Caldera presenta variaciones espaciales en la calidad de las aguas subterrá- 
neas, según se ha observado en estudios realizados en la zona (COP, 1987; Veeger, 1991; König, 1997; Poncela, 2015; CIALP, 2017). En dichos estudios, ha quedado evidenciada la relación de las aguas de baja mineralización muestreadas en manantiales y galerías a cotas altas (dentro y fuera de la Caldera) con el acuífero Coebra (Fig.5). Estas aguas se caracterizan por presentar bajos valores de conductividad eléctri$\mathrm{ca}$, especialmente en el sector Noreste -el de mayor pluviometría- donde se han tomado muestras de solo 70 (CIALP, 2017). La clasificación hidroquímica en base a los iones dominantes da como resultado una mayoría de aguas clasificadas como bicarbonatadas, siendo más variable el contenido catiónico, predominando en primer lugar la composición sódica seguida por una mixta sódico-magnésica o sódico-cálcica (Fig. 6).

König (1997) realiza un análisis factorial, distinguiendo grupos de captaciones en el área de la Caldera, donde la calidad del agua subterránea estaría subordinada a tres factores fundamentales: litología, composición del agua de lluvia y actividad geotermal. El más numeroso de estos grupos conecta varias galerías y manantiales que se localizan aproximadamente entre 900 y 1500 m s. n. m. de altitud, formando un anillo alrededor del borde interno de la Caldera, relacionándolos con las descargas del acuífero Coebra. Otros puntos localizados a cotas inferiores también descargarían aguas del Coebra, pero con una mayor mineralización adquirida a su paso por los

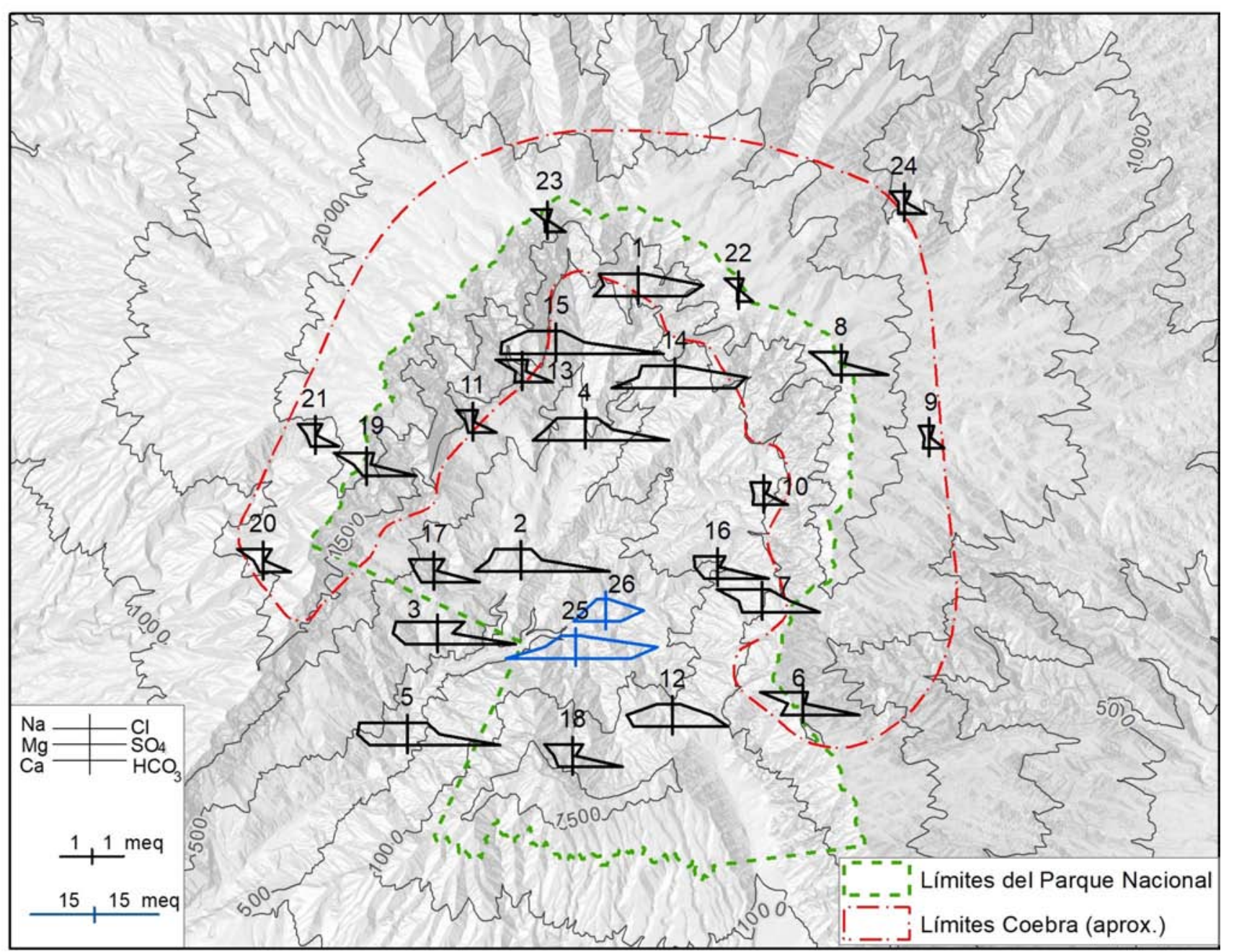

Figura 5. Mapa hidrogeoquímico con diagramas de Stiff-modificado de puntos espacialmente representativos de la zona de estudio. Los datos identificativos de los puntos se muestran en la Tabla 1.

Figure 5. Hydrochemical map with Stiff-modified diagrams of spatially representative points in the study area. (For points data see Table 1). 
Annetty Benavides Gelabert, 2019. La hidrogeología del entorno del Parque Nacional de... Boletín Geológico y Minero, 130 (4): $641-660$

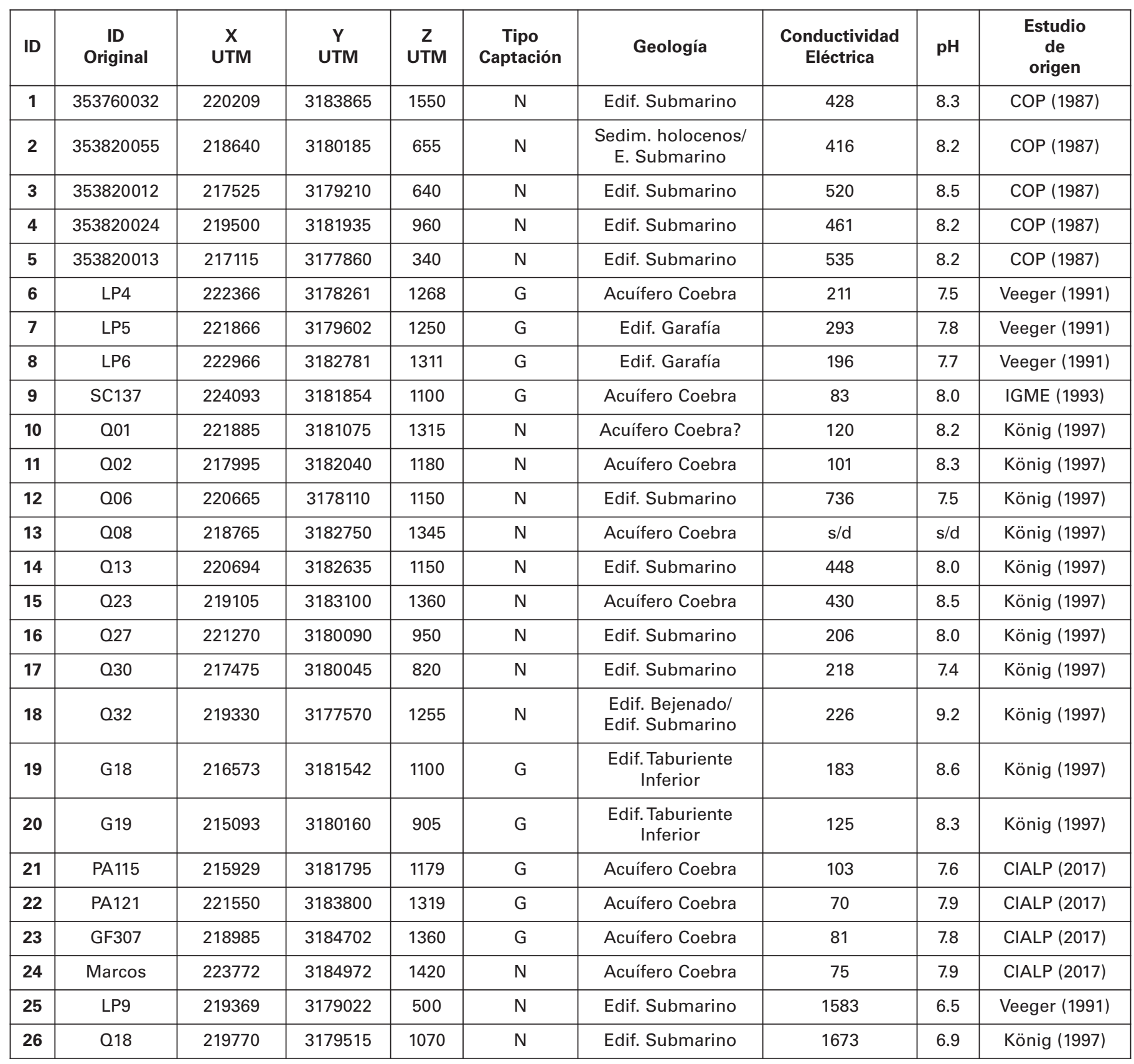

Tabla 1. Datos identificativos de las galerías y nacientes representados en las figuras 5 y 6 , y la geología asociada según los estudios de procedencia (G: Galería; N: Naciente).

Table 1. Data from galleries and springs represented in Figures 5 and 6. The geology of each point has also been referred from previous studies (G: Gallery; N: Spring).

materiales aglomeráticos o sedimentarios de la base del escarpe de la Caldera.

Veeger (1991), König (1997) y Poncela (2015) también coinciden en señalar las particularidades de las aguas muestreadas en manantiales y galerías de la zona baja de la Caldera, en contacto con los materiales pliocenos. Éstas presentan en general temperatu- ras más elevadas (puntualmente superan los $20^{\circ} \mathrm{C}$ ) y mayor concentración iónica que las del acuífero Coebra, alcanzando localmente valores de conductividad eléctrica que superan los 1000 . La composición tiende a ser sulfatada cálcico magnésica, observándose también altos contenidos de hierro disuelto. Veeger (1991) describe aquí un flujo rápido a través 
de una zona geotermal, en el cual la recarga se produciría tanto a través de los materiales sedimentarios de la base del escarpe, como a través de fracturas en los materiales del edificio submarino, dando lugar a una circulación más profunda que descargaría a través de los nacientes del fondo de la Caldera.

König (1997) también señala la mayor concentración de bicarbonatos y sulfatos de las muestras tomadas en manantiales de la zona baja, atribuyéndola a la actividad hidrotermal y a las emanaciones de aníhidrico carbónico $\left(\mathrm{CO}_{2)}\right.$, observando puntualmente valores bajos de $\mathrm{pH}$ que relaciona a la presencia de $\mathrm{CO}_{2}$ disuelto.

Esta diferenciación hidroquímica ha contribuid a establecer la definición de las masas de agua subterráneas en la aplicación de la Directiva Marco del Agua (DGA, 2005; Poncela and Skupien, 2013) delimitándose para la zona baja de la Caldera la masa LP003 o Acuífero del Complejo Basal -de circulación reducida y manantiales de escaso caudal- mientras que el acuífero central o Coebra se incluye dentro de la LP001, Acuífero Insular-Vertientes, considerado el mayor reservorio de agua insular.

\section{Caracterización isotópica}

El estudio de los isótopos estables del agua de La Palma también ha sido abordado en varios de los estudios citados (Veeger, 1991; König, 1997; Poncela, 2015) y por el ITGE (1993). Las rectas meteóricas locales resultantes de estos trabajos difieren entre sí, si bien las condiciones de muestreo de la precipitación y los manantiales seleccionados, son distintos en cada caso, así como la fecha de realización.

En el gráfico de la Figura 7 se representa la relación Oxígeno-18/Deuterio $(\delta 180-\delta \mathrm{D})$ de algunas muestras de aguas subterráneas, cuya localización se muestra en la Figura 5. Puede observarse que se sitúan con bastante aproximación sobre la recta meteórica local de Veeger (1991), elaborada con muestras de 8 manantiales, y sobre la propuesta por Poncela (2015), reconstruida a partir de muestras de manantiales y galerías del período 1991-2015, procedentes de varios estudios y de muestreos específicos llevados a cabo por el propio autor.

En general, las muestras más enriquecidas isotópicamente corresponden a los manantiales de la zona baja de la Caldera, mientras que las más ligeras se relacionan con las muestras de cotas altas, sugiriendo su vinculación al acuífero Coebra. En principio, esta distinción puede atribuirse a las diferentes cotas de recarga, sin embargo, el papel de la orientación de las laderas que rodean la Caldera con respecto a los

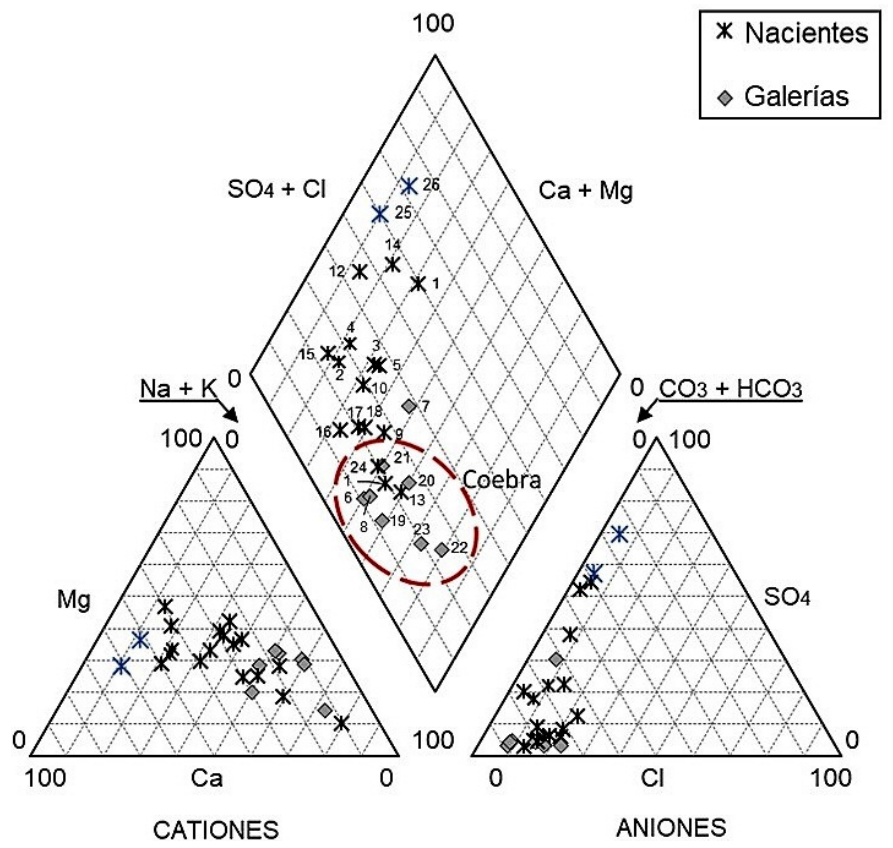

Figura 6. Clasificación hidroquímica según iones dominantes (Diagrama de Pipper) de los puntos representados en la Fig. 5. Figure 6. Hydrochemical classification according to dominant ions (Pipper diagram) referring to points represented in Figure 5.

frentes lluviosos, así como la acción de procesos modificadores en la roca, confieren mayor complejidad a la interpretación.

La relación entre el contenido isotópico y la altitud también pone de manifiesto la delimitación de los acuíferos de la zona en función de la cota de la recarga (Fig. 8). Veeger (1991) calcula una recta altitudinal a partir de muestras de manantiales, en la que no incluye a Marcos y Cordero considerando que la cota de dichos nacientes, de caudal considerable, no es representativa de su cota de recarga, situada a una mayor altitud. Tampoco incluye las muestras con bajo contenido en Tritio, por considerar que podrían introducir grandes discrepancias entre la cota de muestreo y la de recarga, incluyendo sólo las que presentaban un alto contenido de este isótopo por ser las representativas de aguas de recarga reciente.

Dentro de los datos disponibles, no se han identificado muestras que sigan estos criterios, por lo que se han mantenido las rectas originales propuestas por dicha autora, a pesar de que algunos valores de oxígeno-18 quedan algo desplazados por encima de esta recta, lo que puede ser consecuencia de cotas de recarga y descarga muy próximas.

Las muestras localizadas en la base de la Caldera reflejan cotas de recarga variables, sin superar los 1400 m s.n.m. En cambio, las relacionadas al acuífero 


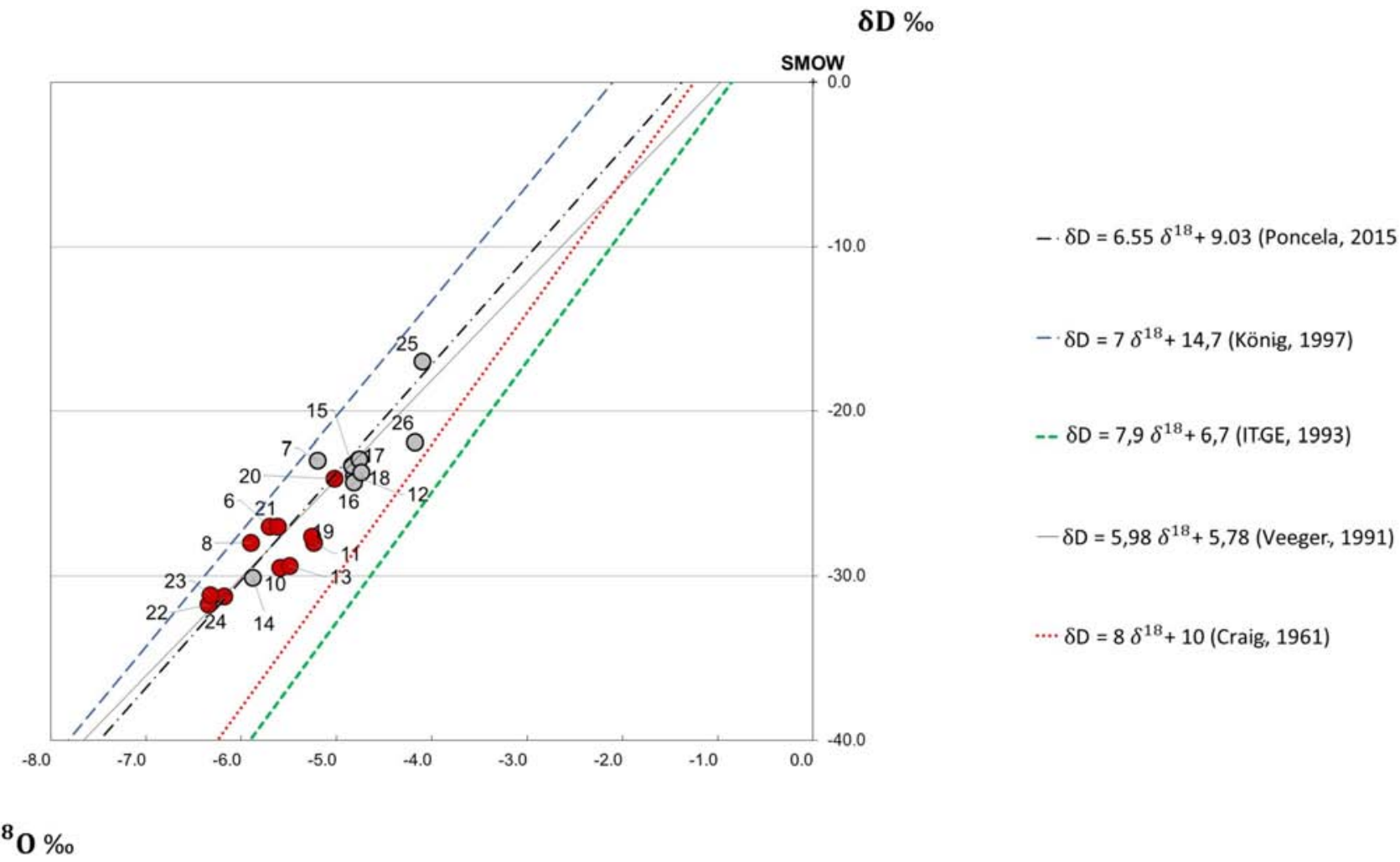

Figura 7. Relación en aguas subterráneas de la zona de estudio. En rojo, las muestras asociadas al acuífero Coebra (véase Fig. 5 y Tab. 1 para localización de los puntos).

Figure 7. Relation in groundwater from the study area. Samples associated to Coebra aquifer are marked in red (see Fig.5 and Table 1 for location of the points).

Coebra, sugieren cotas de recarga más elevadas. Los valores más altos se registran en las captaciones situadas en el sector N-NE (galerías 22 y 23 , y naciente Marcos, 24), con cotas de recarga próximas a los 2000 m s. n. m., lo que también ha sido comprobado por Skupien y Poncela (2011).

Por otra parte, las muestras 6 y 8 , en el brazo oriental del acuífero Coebra, así como la 11, 19 y 20, en el brazo occidental, presentan cotas de recarga que oscilan entre 1400 y 1650 m s. n. m. Teniendo en cuenta la topografía local, esto reafirmaría el predominio de la permeabilidad vertical dentro del propio acuífero Coebra, donde cada sector se alimentaría fundamentalmente de la lluvia caída en su vertical.

Cabe mencionar que algunas galerías situadas a altitudes medias, en las vertientes exteriores del cono norte, también refieren cotas de recarga muy elevadas (ITGE, 1993; CIALP, 2017), sólo posibles en el área del acuífero Coebra, lo que corrobora la existencia del flujo radial regional, aunque limitado por zonas en función del límite subterráneo de la estructura Coebra, la permeabilidad horizontal y la magnitud de la recarga.
Las consideraciones previas, realizadas a partir del contenido de isótopos estables, presentan la limitación de haber sido tomadas en condiciones y fechas dispares. No obstante, constituyen una muestra de cómo las determinaciones de isótopos estables, de relativo bajo coste económico, aportan información significativa para entender las condiciones de la recarga y el funcionamiento de los acuíferos insulares, tal como demuestran recientes estudios realizados en otras islas de Canarias (Marrero, 2010; Hernández-Quesada, 2015).

Con relación al análisis del contenido de Tritio, algunas determinaciones recientes realizadas en la zona (CIALP, 2017) pueden ser interpretadas según el modelo propuesto por Poncela (2015) para La Palma. Este modelo, cuyo año de referencia es 2014, establece dos sistemas de flujo subterráneo: uno regional de circulación lenta y profundo que se correspondería con valores inferiores a $0,5 \mathrm{UT}$, indicativos de tiempos de tránsito de edad pre-nuclear con largos tiempos de residencia y poco aporte de agua reciente; y otro de flujos locales, de recarga reciente, rápida circulación y poca mineralización, que presentarían valores de 

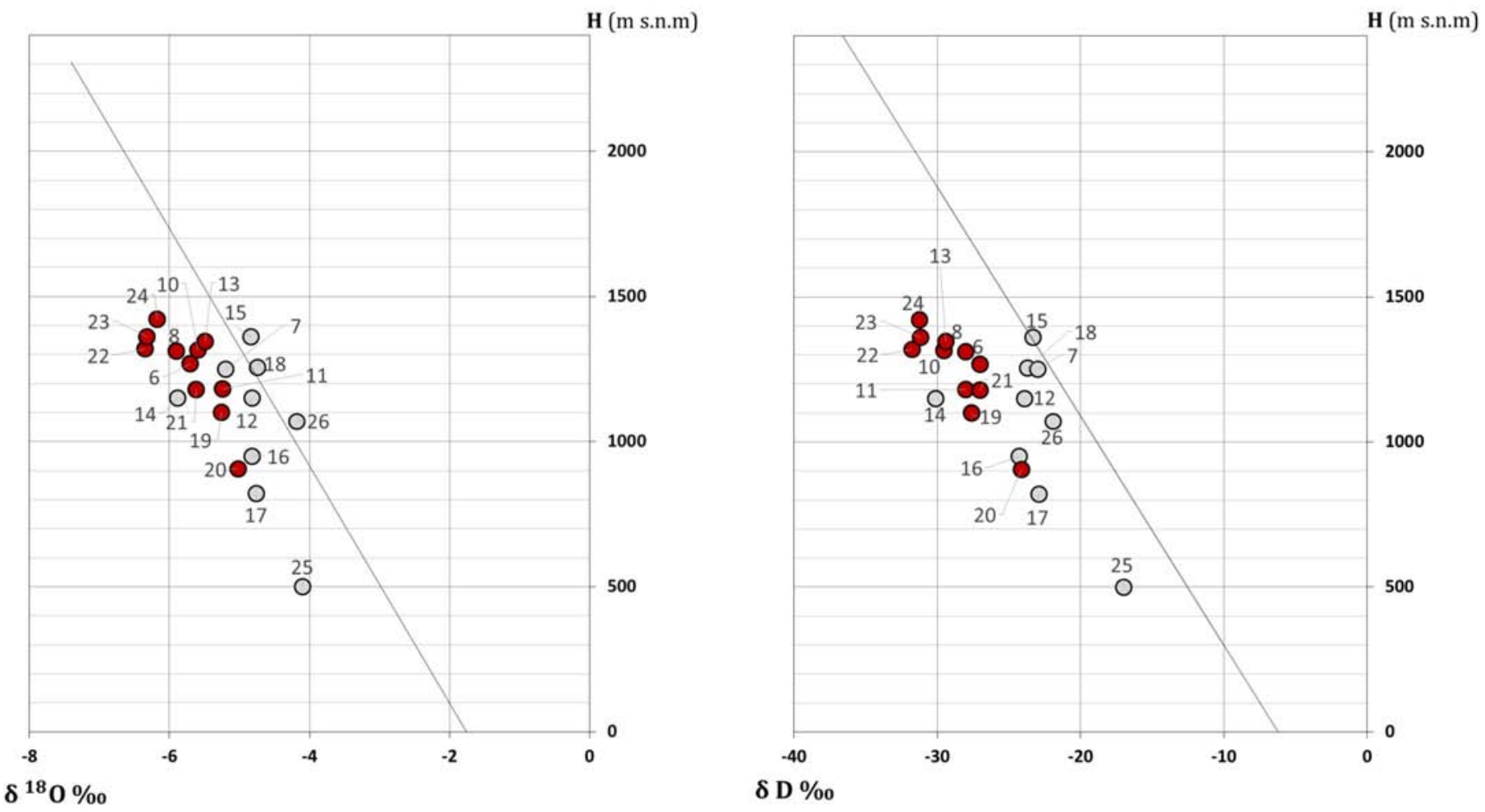

Figura 8. Estimación de cotas de recarga a partir de las rectas vs Altitud de Veeger (1991).

Figure 8. Estimation of recharge elevations from vs Elevation Veeger's line (1991).

Tritio contrastables en las aguas subterráneas $(>1,5-2$ UT). Valores entre 0,5 y 1,5 UT se consideran situaciones intermedias.

El mayor de los valores medidos (1,2 UT) corresponde a la galería 23, cuya principal zona de aporte se localiza a una cota elevada (1600 m s. n. m.) lo que, además de la baja conductividad eléctrica (81), permite interpretar que corresponde al tipo de flujo local con aguas recientes recargadas en las zonas de mayor altitud dentro del sector Coebra.

En el naciente Marcos, Skupien y Poncela (2011) obtienen en 2008 un valor de 1,6 UT, siendo interpretado como un claro indicio de recarga reciente a partir de precipitaciones actuales en la zona de cumbre, a cotas muy superiores a la de descarga. Por su parte, Veeger (1991) atribuye el alto contenido de Tritio que obtiene en 1990 (5,9 UT) a las altas velocidades del flujo de agua subterránea hacia el naciente, recargadas a altitudes muy superiores a la de muestreo. El contenido actual de Tritio (1 UT) lo situaría en las situaciones intermedias que propone el citado modelo, aunque dado el año de referencia del mismo (2014) en la actualidad este valor podría interpretarse propio de aguas de recarga reciente.

Por último, en la muestra 22 , correspondiente a una galería que explota el acuífero Coebra, se obtiene 0,9 UT (CE=79). Este valor presenta una situación similar al naciente Marcos, donde probablemente la proporción de aguas de recarga reciente sea mayoritaria, si bien para esta misma galería, Veeger (1991) reporta un valor de 2,2 UT ( $C E=87$ ) interpretando en aquel contexto que este valor indicaba largos tiempos de tránsito desde la zona de recarga a la de muestreo.

Con todo, a partir de las determinaciones de Tritio realizadas y recopiladas, y teniendo en cuenta el modelo propuesto por Poncela (2015), puede resumirse que en las cotas más altas del cono norte, coincidentes con el sector Coebra y sus laderas exteriores, las aguas subterráneas, a juzgar por su contenido deTritio, son de recarga bastante reciente.

\section{Balance hidrometeorológico del acuífero Coebra}

Los planes hidrológicos insulares realizados hasta la fecha han incluido cálculos de balance hidrometeorológico para la isla basados en los datos de estaciones meteorológicas existentes, sobre todo a cotas medias y bajas. El documento más reciente calcula para toda 
la isla valores de precipitación (737 mm), evapotranspiración real $(353 \mathrm{~mm}$ ) y estima la escorrentía superficial $(23 \mathrm{~mm})$ resultando una recarga media de 361 $\mathrm{mm} / \mathrm{año}$ de los cuales más del $85 \%$ corresponderían a los acuíferos del cono norte insular.

En particular, la estimación del balance hidrometeorológico de la zona de cumbres -con la mayor elevación insular (Roque de Los Muchachos, $2426 \mathrm{~m}$ ) y área de recarga del acuífero Coebra- conlleva dificultades debido a la ausencia de estaciones meteorológicas que proporcionen series termométricas o pluviométricas extensas. En un reciente trabajo realizado por la autora en el marco de una asistencia técnica para el Consejo Insular de Aguas de La Palma (CIALP, 2017) se realiza una aproximación a este cálculo partir de las series mensuales disponibles (AEMET), y de la reconstrucción parcial de las series de la estación C140U (Roque de Los Muchachos) con datos pluviométricos y termométricos facilitados por el Grupo de Calidad Astronómica del Cielo y el Telescopio NOT, respectivamente, ambos pertenecientes al Instituto de Astrofísica de Canarias.

Los límites del sistema, si bien es difícil definir con exactitud la extensión subterránea de la estructura Coebra, a efectos de su estudio se ha determinado la superficie a partir de la cartografía geológica (véase Fig.4), resultando unos $38 \mathrm{~km}^{2}$. En cuanto a los límites temporales, se ha utilizado el período 1985-2015 expresado en años hidrológicos (OctubreSeptiembre).

El relleno de las series se ha abordado mediante el análisis correlacional siguiendo la metodología empleada por Heredia y Meléndez (2016) para la subcuenca del río Pas en Puente Viesgo (Cantabria), con el objetivo de rellenar los huecos existentes y conseguir series completas que permitieran el cómputo de la pluviometría y la evapotranspiración, parámetros fundamentales para el cálculo del balance hidrometeorológico. La selección de las series pluviométricas y termométricas usadas en el análisis correlacional se realiza en base a un mínimo de $60 \%$ de datos disponibles para el período mencionado, sin embargo, se seleccionaron puntualmente estaciones con menor cantidad de datos en áreas geográficas de interés para la zona de estudio (Fig. 9).

Para el relleno se eligen las series de referencia

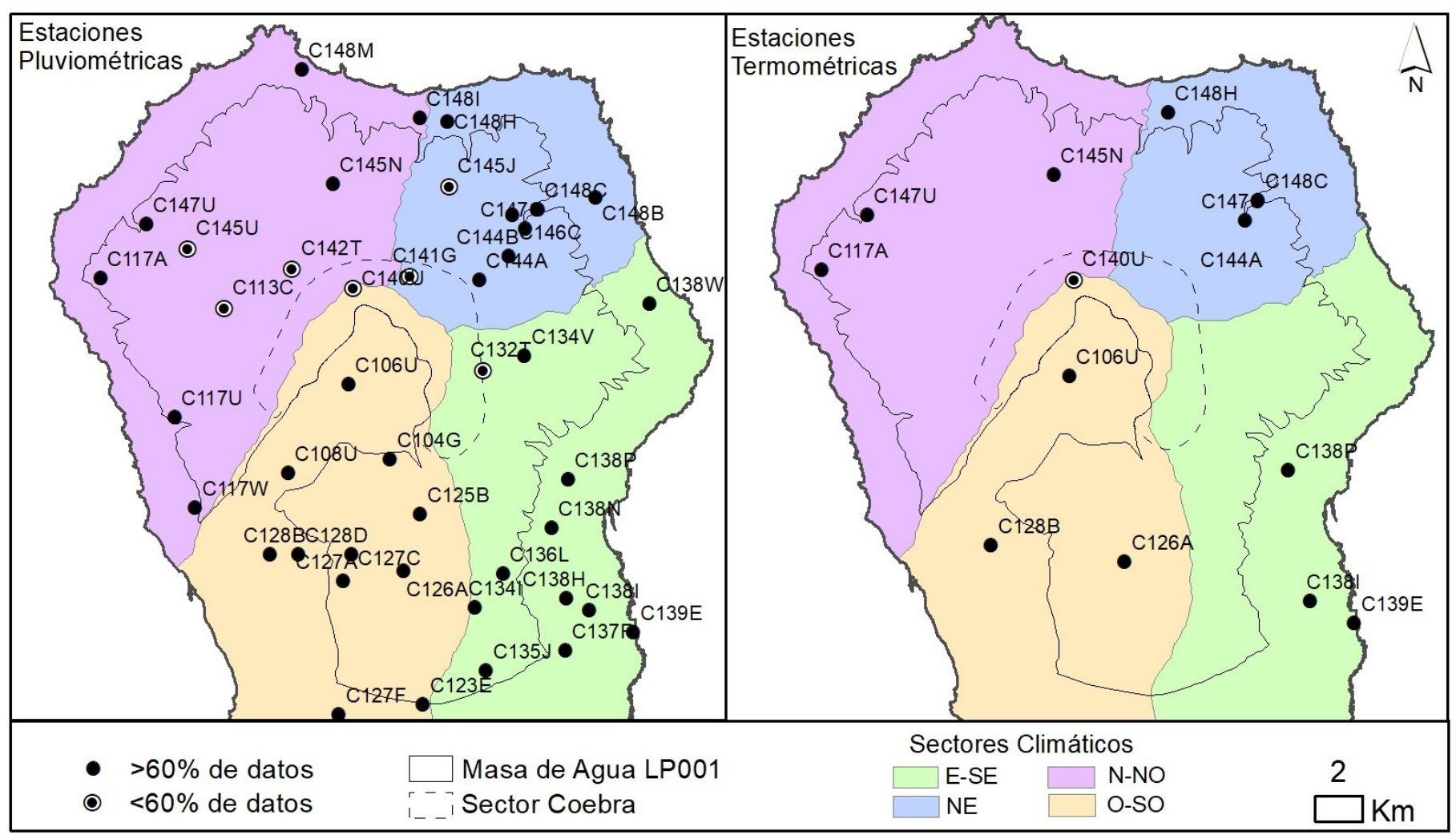

Figura 9. Estaciones meteorológicas (AEMET) empleadas en el análisis correlacional. Los sectores climáticos son los definidos en CLIMCAN-010 (2010).

Figure 9. Meteorological stations (AEMET) used in correlational analysis. Climatic sectors as defined in CLIMCAN-010 (2010). 
con la mayor correlación $(R)$ con la serie a rellenar. Dado que en ocasiones se trataba de estaciones muy alejadas geográficamente y dadas las peculiaridades de la geografía de La Palma donde la orientación de las vertientes influye notablemente sobre las variables climáticas, se añadieron otros criterios:

Alta correlación $(R>0,8)$ entre la serie a rellenar y la serie de referencia.

Las estaciones deben encontrarse en el mismo sector climático (CLIMCAN, 2010).

Si la mayor correlación se presenta entre series de distintos sectores climáticos, la distancia entre ambas no deberá superar los $5 \mathrm{~km}$.

En las Tablas 2 y 3 se indica el proceso secuencial del relleno, las estaciones que intervienen, los respectivos coeficientes de correlación, los meses sin datos, los remanentes después de cada relleno así como los valores medios mensuales antes y después del relleno. Según los criterios establecidos, las correlaciones significativas son bastante altas y se considera que el promedio final de las series rellenas es aceptable dada la insuficiencia de datos, la escala

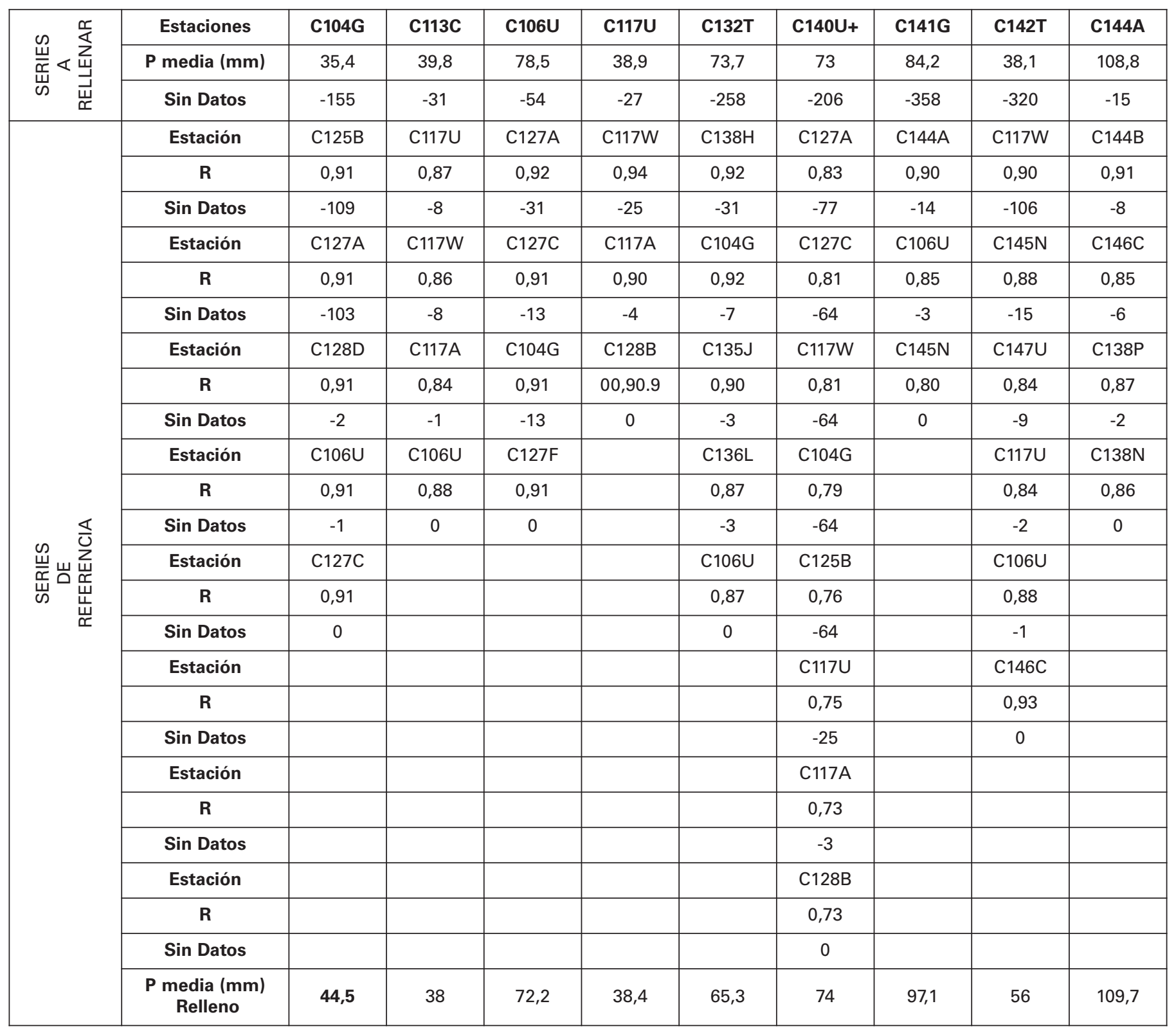

Tabla 2. Secuencia del relleno de las series pluviométricas.

Table 2. Sequence of rainfall series filling. 


\begin{tabular}{|c|c|c|c|}
\hline \multirow{3}{*}{ 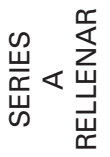 } & Estaciones & C106U & C140U+ \\
\hline & $\mathrm{T}$ media $\left({ }^{\circ} \mathrm{C}\right)$ & 15,9 & 8,6 \\
\hline & S/D & 37 & 16 \\
\hline \multirow{10}{*}{ 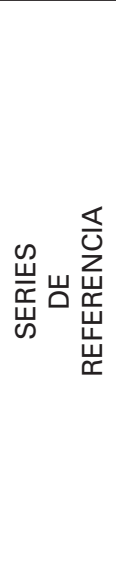 } & Est. & C126A & $\mathrm{C} 106 \mathrm{U}$ \\
\hline & $\mathbf{R}$ & 0,94 & 0,85 \\
\hline & S/D & 13 & 3 \\
\hline & Est. & C117A & C117A \\
\hline & $\mathbf{R}$ & 0,93 & 0,87 \\
\hline & S/D & 3 & 3 \\
\hline & Est. & C147U & C147U \\
\hline & $\mathbf{R}$ & 0,92 & 0,89 \\
\hline & S/D & 0 & 0 \\
\hline & $\begin{array}{c}\mathrm{T} \text { media }\left({ }^{\circ} \mathrm{C}\right) \\
\text { Relleno }\end{array}$ & 15,9 & 8,6 \\
\hline
\end{tabular}

Tabla 3. Secuencia del relleno de las series termométricas. Table 3. Sequence of temperature series filling.

mensual y el método estadístico empleado para el relleno.

\section{Cálculo zonal de las precipitaciones}

El cálculo zonal de la precipitación media mensual (mm) para el período considerado (1985-2015) se realiza por el método de los polígonos de Thiessen, proyectando las áreas de influencia de cada estación sobre la zona de estudio, considerando proporcional la aportación de cada valor medio mensual al área que ocupa. En la Fig.10 se presentan las estaciones pluviométricas involucradas y la superficie de los polígonos que se inscriben en la zona, así como el porcentaje de contribución de los mismos al cálculo de la $\mathrm{P}$ media mensual de todo el período.

Como resultado, se ha computado en el área del Coebra un valor de precipitación media anual de 917 $\mathrm{mm}$, con un mínimo de $165 \mathrm{~mm}$ para el año hidrológico 1994-1995 y un máximo de $1850 \mathrm{~mm}$ para 20042005. Si se consideran escenarios climáticos secos y húmedos en base a una desviación de un $20 \%$ con respecto a la media, resultan valores de $580 \mathrm{~mm}$ y $1372 \mathrm{~mm}$ respectivamente, superiores a los de ámbito insular (APHP, 2015) donde el valor medio anual se establece en unos $737 \mathrm{~mm}$, oscilando entre los 488 $\mathrm{mm}$ para los años secos y $986 \mathrm{~mm}$ para húmedos. En efecto, la zona de estudio se encuentra dentro de los mayores relieves insulares, muy influenciada por los vientos alisios -particularmente la parte NE- responsables de la mayor parte de la pluviometría y recarga locales.

\section{Cálculo zonal de la temperatura y evapotranspiración potencial}

El cálculo zonal de las temperaturas medias, mínimas y máximas mensuales se ha realizado, al igual que la pluviometría, por el método de los polígonos de Thiessen. Dada la escasez de estaciones termométricas en la zona, y conocida la estrecha relación entre la temperatura y la altitud en las islas del archipiélago, se han introducido algunas series virtuales siguiendo la metodología de Heredia y Meléndez (2016), considerando que las estaciones con series disponibles, localizadas a menor altitud, proyectarían valores erróneos de temperatura sobre el área de estudio.

Los altos coeficientes de determinación resultantes del análisis correlacional pone de manifiesto la relación entre estas dos variables en la zona de estudio (Fig. 11). La serie termométrica mensual de cada estación virtual se ha calculado mediante la media ponderada de los valores de temperatura mensual de las estaciones participantes, donde el correspondiente factor de ponderación es la relación entre la temperatura media anual estimada de la estación virtual y la temperatura media anual de cada estación de referencia.

En la Figura 12 se presentan las estaciones termométricas involucradas y la superficie que ocupan los polígonos en la zona de estudio, señalando las estaciones virtuales, situadas en la misma localización de estaciones pluviométricas que carecen de datos de temperatura.

El cálculo de la evapotranspiración potencial, y ante la ausencia de datos para la aplicación de la formulación de Penman-Monteih recomendada por la FAO (Allen et al., 2006), se ha abordado mediante el método clásico de Thornthwaite (1948) para determinar la ETP, y por la fórmula simplificada de Hargreaves (Hargreaves and Samani, 1985), asimilando la $\mathrm{ET}_{0}$ a la $\mathrm{ET}$ con un factor de bosque $\mathrm{Kc}=1$.

En los resultados obtenidos, los valores de la ETP superan a la $\mathrm{ET}_{0}$ en todos los ciclos hidrológicos considerados, siendo la diferencia del $27 \%$, lo que demuestra la importancia de la elección del método de estimación de la evapotranspiración para el cálculo del balance del agua en el suelo. No obstante, cabe mencionar la incertidumbre que presenta la estimación de esta variable, dado el tratamiento de estadístico realizado, siendo necesaria la introducción de series termométricas virtuales. 


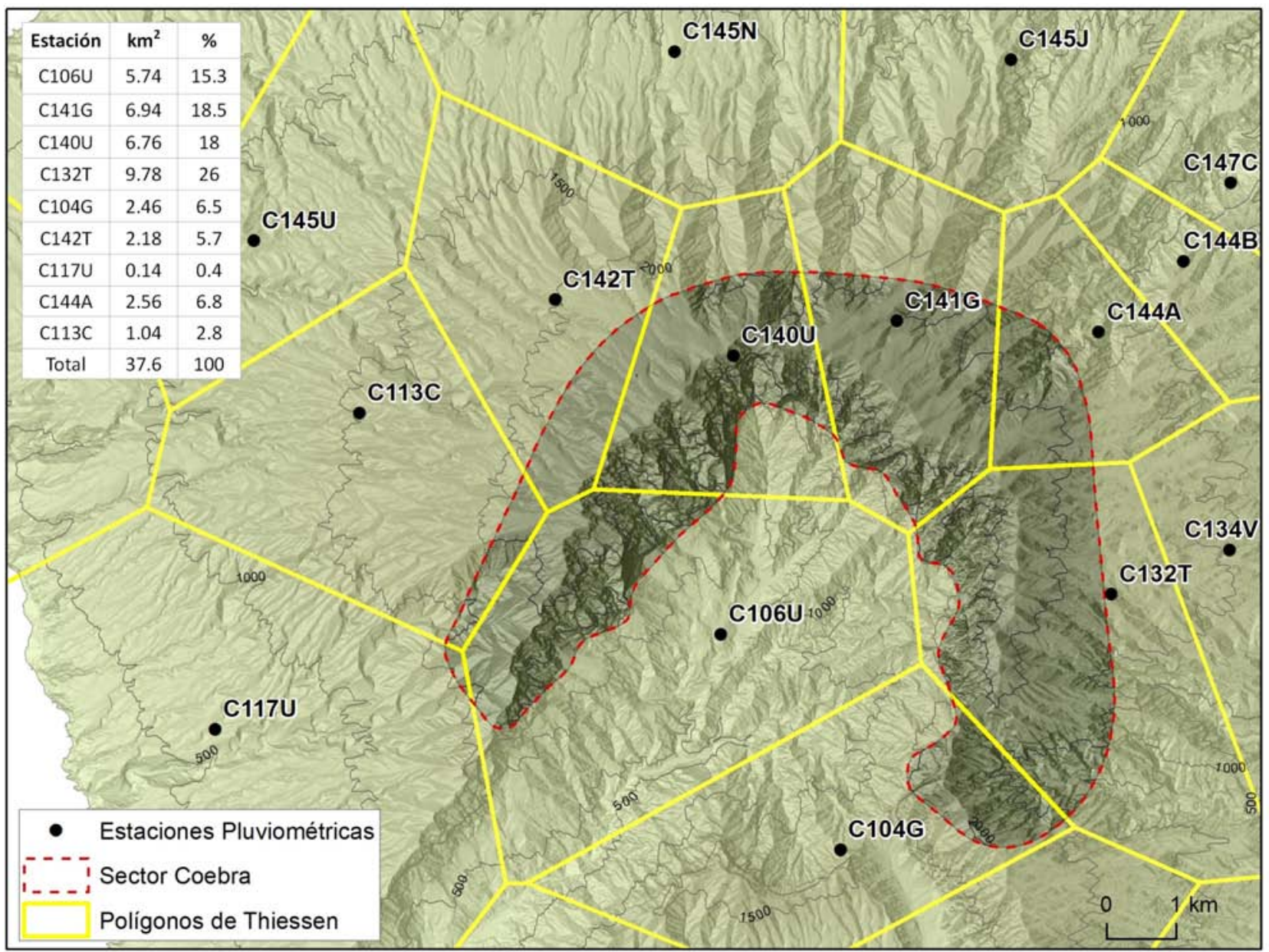

Figura 10. Cálculo zonal de la precipitación media mensual del área del acuífero Coebra mediante el método de los polígonos de Thiessen (1985-2015).

Figure 10. Zonal calculation of average monthly rainfall in Coebra aquifer area using the Thiessen polygons method (1985-2015).
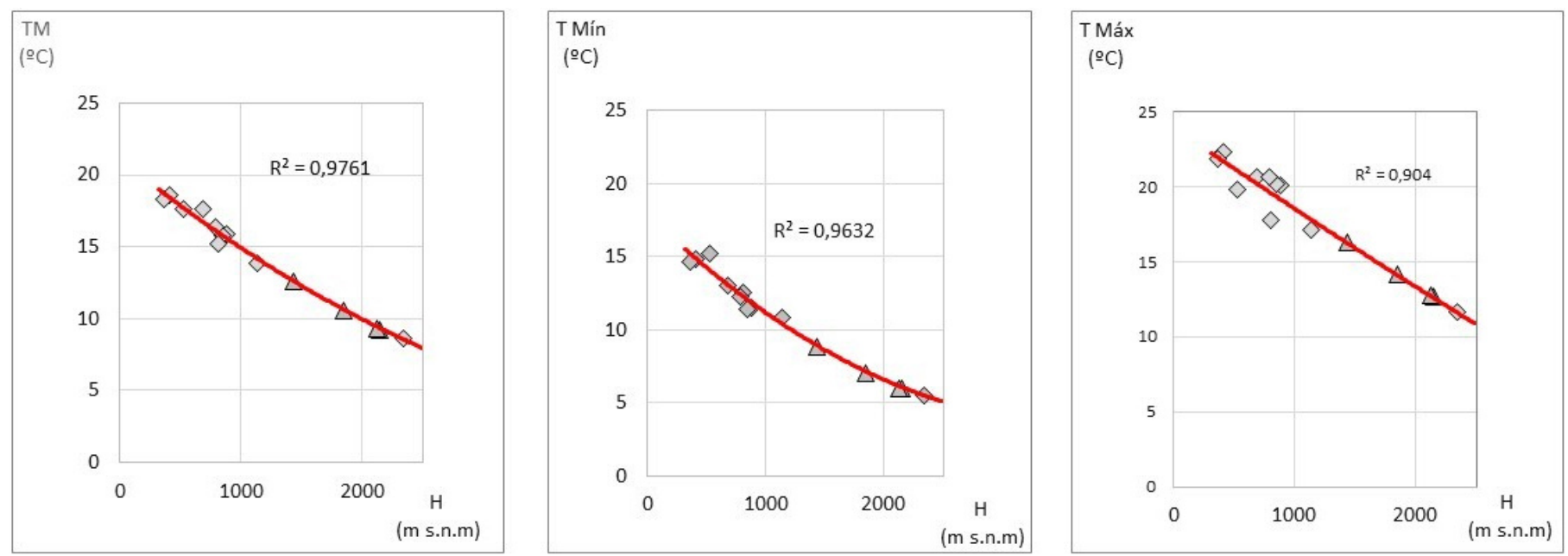

Figura 11. Análisis correlacional entre cota y temperatura (media, mínima y máxima anual) de las estaciones termométricas seleccionadas para el análisis. Las estaciones virtuales se representan con triángulos.

Figure 11. Correlation analysis between elevation and temperature (mean, minimum and maximum annual) of thermometric stations selected for the analysis. Virtual stations are represented by triangles. 


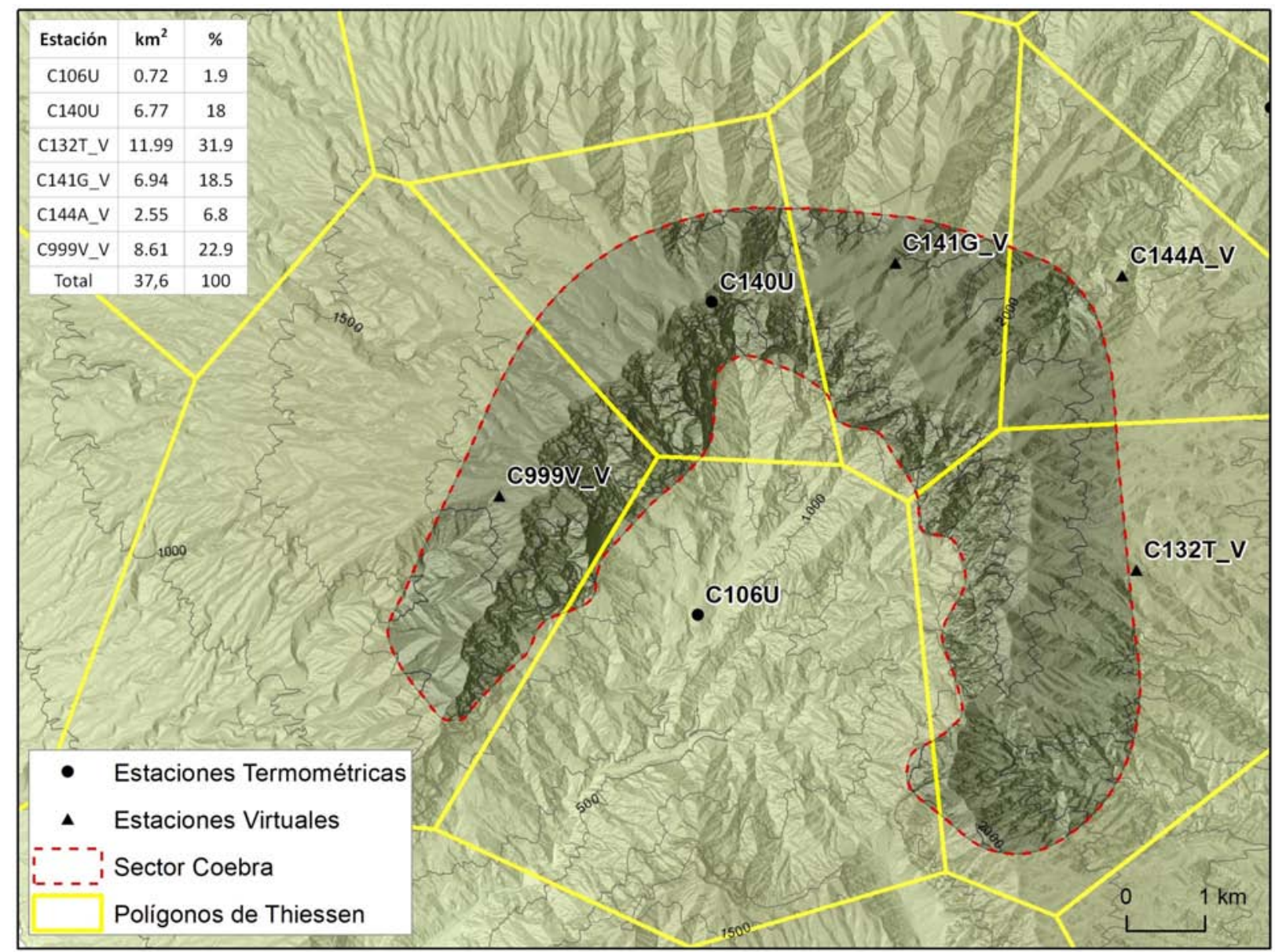

Figura 12. Cálculo zonal de la temperatura (media, mínima y máxima mensual) del área del acuífero Coebra mediante el método de los polígonos de Thiessen.

Figure 12. Zonal calculation of temperature (monthly mean, minimum and maximum) in Coebra aquifer area using the Thiessen polygons method (1985-2015).

\section{Estimación del balance hidrometeorológico en el acuífero Coebra}

En la Tabla 4 se resumen varias propuestas de balance hidrometeorológico para el período 1985-2015, representado como valores medios anuales de los 30 ciclos hidrológicos considerados.

Se han empleado los valores calculados de ETP y $\mathrm{ET}_{0}$, obtenidos según los métodos de Thornthwaite (1948) y Hargreaves y Samani (1985), respectivamente, para el posterior cálculo de la evapotranspiración real (ETR). A falta de parámetros edáficos de la zona de estudio, se ha usado un valor típico de reserva de agua utilizable por las plantas $(50 \mathrm{~mm})$, empleando en segundo lugar un valor inferior $(10 \mathrm{~mm})$, considerando los materiales geológicos descritos en la zona, el poco desarrollo de los suelos y el tipo de vegetación presente.

La estimación de la escorrentía directa se ha abor- dado mediante el método empírico del National Resource Conservation Service de Estados Unidos (NRCS, 2004) que utiliza valores tabulados del umbral de escorrentía $\left(P_{o}\right)$ en función del tipo de suelo. Para la zona de estudio, según la superficie ocupada por cada tipo de suelo (Corine Land Cover) y los valores de $P_{0}$ tabulados en la Norma 5.2 IC (Ministerio de Fomento, 2016), se ha estimado una media ponderada de $P_{o}=58$. Con este método se ha obtenido una escorrentía directa que representa entre el $10 \%$ y el $56 \%$ de la precipitación dependiendo del año hidrológico -con excepción de dos años en los que no se registra escorrentía directa- siendo la media del período del $25 \%$. Este valor, aunque estimado, es coherente con el propuesto en el SPA-15 (1975) para el área de estudio, donde se estima un valor de escorrentía superficial del $20 \%$ de la precipitación, y del 50 $\%$ para zonas interiores de la Caldera.

El cálculo del excedente varía en función de los 
Annetty Benavides Gelabert, 2019. La hidrogeología del entorno del Parque Nacional de... Boletín Geológico y Minero, 130 (4): $641-660$

\begin{tabular}{|c|c|c|c|c|c|c|c|c|}
\hline & $\begin{array}{l}\text { Precipitación } \\
\text { mm }\end{array}$ & $\begin{array}{l}\text { Escorrentía } \\
\text { Directa } \\
\text { mm }\end{array}$ & $\begin{array}{c}\text { Reserva } \\
\text { Útil } \\
\text { mm }\end{array}$ & $\begin{array}{l}\text { Método de } \\
\text { cálculo } \\
\text { ETP/ET }_{0}\end{array}$ & $\begin{array}{l}\text { ETP/ET }_{0} \\
\text { mm }\end{array}$ & $\begin{array}{l}\text { ETR } \\
\text { mm }\end{array}$ & $\begin{array}{l}\text { Excedente } \\
\mathbf{m m}\end{array}$ & $\begin{array}{c}\text { Infiltración } \\
\text { Estimada } \\
\text { mm }\end{array}$ \\
\hline \multirow{3}{*}{$\begin{array}{l}\text { Media Anual } \\
\text { 1985-2015 }\end{array}$} & \multirow{3}{*}{917} & \multirow{3}{*}{262} & \multirow{2}{*}{50} & Tornthwaite & 595 & 302 & 624 & 362 \\
\hline & & & & Hargreaves & 817 & 361 & 557 & 295 \\
\hline & & & 10 & Tornthwaite & 595 & 265 & 661 & 400 \\
\hline
\end{tabular}

Tabla 4. Propuestas del balance hidrometeorológico del sector Coebra (valores medios anuales para el período 1985-2015) según diferentes métodos de estimación de la evapotranspiración (ETP/ET $)$ y valores típicos de reserva de agua útil de 10 y $50 \mathrm{~mm}$. La escorrentía se ha estimado por el método del NRCS (2004).

Table 4. Proposals for the hydrometeorological balance of the Coebra sector (annual average values for the period 1985-2015) according to different estimation methods of evapotranspiration (ETP/ET $T_{0}$ and typical values of extractable soil water (10/50 mm). Runoff has been estimated by the NRCS method (2004).

valores de partida, observándose diferencias de algunas decenas de milímetros según el año hidrológico. El valor obtenido empleando la ETP es del $8 \%$ superior respecto al calculado con la $E T_{0}$ para $R U=50 \mathrm{~mm}$, y de $11 \%$ para $R U=10 \mathrm{~mm}$. Esta diferencia repercute directamente en la posterior estimación de la recarga, hecho ya observado a nivel insular $\left(706 \mathrm{~km}^{2}\right)$ por Poncela (2015), planteando una infravaloración del valor de la ETP calculada mediante Thornthwaite, y la consecuente sobreestimación de la infiltración.

No obstante, a pesar de las incertidumbres que presentan los resultados propuestos, estos proporcionan una primera aproximación al valor de la recarga natural del acuífero Coebra, a falta de parámetros necesarios para la aplicación de métodos más precisos como el de Penman-Monteith, no disponibles en la actualidad.

\section{Conclusiones}

Estudios hidrogeológicos previos realizados en el entorno del Parque Nacional de la Caldera de Taburiente, demuestran la delimitación de dos áreas de circulación y calidad de las aguas subterráneas en la zona. Los manantiales y galerías situados a elevadas cotas (>1000 m s.n.m.), tanto en la pared interior de la Caldera como en el borde exterior, se relacionan al acuífero Coebra, emplazado sobre una discordancia erosiva asociada a un gran deslizamiento. Las aguas de este acuífero presentan muy baja mineralización y una composición predominantemente bicarbonatada sódica, mientras que las aguas subterráneas asociadas a los materiales pliocenos de la zona deprimida de la Caldera, con caudales más reducidos, presentan mayor mineralización, alta concentración de bicarbonatos y sulfatos, estando localmente influenciados por la actividad geotermal.
El análisis basado en el contenido de isótopos estables también muestra esta diferenciación, reflejando unas cotas de recarga más elevadas para el acuífero Coebra, así como una gran vinculación con la pluviometría local según orientación de las vertientes y predominio de la permeabilidad vertical, favorecida por las numerosas intrusiones de diques. No obstante, el flujo subterráneo dentro y a través de los límites de este acuífero, así como la extensión subterránea de la base impermeable y el funcionamiento hidráulico del sistema, presentan aún muchas interrogantes.

Se presentan varias propuestas de cálculo del balance hidrometeorológico del área del acuífero Coebra, según diferentes datos de partida, constituyendo una primera aproximación de este cálculo en la zona. Estos resultados, no obstante, presentan incertidumbres debido, en primer lugar, a la escasez de series pluviométricas y termométricas en la zona de estudio, razón por la cual se ha empleado un método estadístico para el relleno de series pluviométricas y termométricas mensuales muy incompletas y se han introducido estaciones virtuales en zonas desprovistas de mediciones.

Los términos naturales del balance se han estimado mediante métodos de cálculo empíricos. Así, la escorrentía superficial se ha estimado a partir del método del National Resource Conservation Service de Estados Unidos (NRCS, 2004) obteniéndose un valor medio anual del $25 \%$ de la precipitación. El cálculo de la evapotranspiración se ha abordado mediante dos métodos -Thornthwaite (1948) y Hargreaves and Samani (1985)- obteniéndose resultados variables, con una diferencia de aproximadamente un $27 \%$, lo que influye en el posterior cálculo del excedente, y consecuentemente en la estimación de la infiltración y recarga.

Estos resultados, a pesar de las incertidumbres 
que contienen, representan un avance en la estimación del balance hídrico de la zona, en tanto no se disponga de un mejor ajuste mediante métodos como el de Penman-Monteith. En el futuro, y con vistas a la correcta gestión de los recursos hídricos del entorno del Parque Nacional de la Caldera de Taburiente y de la isla de La Palma, estos resultados deberán ser ajustados mediante la obtención de mediciones específicas de la zona, tanto de parámetros de suelo, como de datos meteorológicos diarios que proporcionen series robustas, a lo que sin duda contribuiría el proyecto de implantación de una agencia de meteorología canaria.

\section{Agradecimientos}

El presente trabajo ha sido realizado gracias al proyecto promovido y financiado por el Consejo Insular de Aguas de La Palma, Estudio Hidrogeológico sobre la estimación de la recarga natural del acuífero Coebra y su posible sobreexplotación zonal dentro de la Masa de Agua LP0001, La Palma, Islas Canarias.

La autora también desea expresar su agradecimiento a las instituciones cuyas contribuciones han sido imprescindibles para el desarrollo de este trabajo: Instituto Geológico y Minero de España (IGME), Agencia Estatal de Meteorología (AEMET), Nordic Optical Telescope (NOT) y Grupo de Calidad Astronómica del Cielo (Instituto Astrofísica de Canarias). Gracias también a los colegas hidrogeólogos consultados, cuyas observaciones han sido de incalculable ayuda.

\section{Referencias}

Allen, R.; Pereira, L.; Raes, D.; Smith, M. 2006. Evapotranspiración del cultivo: guías para la determinación de los requerimientos de agua de los cultivos. Estudio FAO Riego y Drenaje Vol. 56. ISSN 0254-5293

APHP. 2015. Texto refundido de la normas de ordenación parcial del Plan Hidrológico de la Demarcación Hidrográfica de La Palma. Consejo Insular de Aguas de La Palma.

Bellido Mulas, F, Gómez Sainz de Aja, J.A., \& Barrera, J.L. 2004. Mapa Geológico Digital continuo E. 1:25000, Zona Canarias-La Palma. Zona-2915). Recuperado el 8 de 12 de 2016, de IGME: http://info.igme.es/cartografiadigital/datos/geodezona.aspx?ld=Z2915

Bravo, T., \& Coello, J. 1979. Contribución a la hidrogeología de la Caldera de Taburiente, La Palma, Islas Canarias. II Simposio Nacional de Hidrogeología. Pamplona, 525-532.

Buch, L. v. 1825. Descripción física de las Islas Canarias. La Orotava ( $\mathrm{S} / \mathrm{C}$ de Tenerife) (trad. castellana de la ed. francesa 1836), $263 \mathrm{pp}$.
Cabrera, M., and Custodio, E. 2011. La investigación hidrogeológica en Canarias desde el proyecto Canarias SPA15. In: Cabrera, M. y Custodio, E. (ed.), El conocimiento de los recursos hídricos en Canarias cuatro décadas después del proyecto SPA-15. ISBN: 978-84-938046-0-2. Las Palmas de Gran Canaria,109-116.

Carracedo, J., Badiola, J., and Guillou, H. 2015. Mapa Geológico de España escala 1:25000. Vols. (Hojas 1083-I a 1083-IV). Madrid: Instituto Geológico y Minero de España. ISBN: 978-84-7840-978-5.

CIALP. 2017. Estudio Hidrogeológico sobre la estimación de la recarga natural del acuífero Coebra y su posible sobreexplotación zonal dentro de la Masa de Agua LP001. La Palma, Islas Canarias. Informe Inédito. Consejo Insular de Aguas de La Palma.

CLIMCAN-010. 2010. Caracterización climática de las Islas Canarias para la aplicacion del Código Técnico de la Edificación. Gobierno de Canarias.

Coello Armenta, J. 1987. Las aguas subterráneas en las formaciones volcánicas del norte de La Palma (Islas Canarias). Simposio Internacional de Recursos Hidráulicos CANARIAS 2000. Consejería de Obras Públicas. Gobierno de Canarias.

COP. 1987. Estudio Hidrológico, Hidrogeológico y Geohidroquímico con inventario de puntos de agua de La Caldera de Taburiente. Consejería de Obras Públicas. Gobierno de Canarias.

Custodio, E., and Cabrera, M.C. 2008. Síntesis de la Hidrogeología de las Islas Canarias. Geo-Temas, 785788.

Custodio, E., and Llamas, M.R. 1987. Hidrología Subterránea 2001 ed., Vol. I). Barcelona: Omega.

DGA. Dirección General de Aguas. Gobierno de Canarias. 2005. Actividades requeridas para el cumplimiento de la Directiva Marco del Agua en las islas de Tenerife, La Palma, La Gomera y El Hierro. Consejería de Infraestructuras, Transportes y Viviendas. Gobierno de Canarias, Dirección General de Aguas.

Hargreaves, G., and Samani, Z. 1985. Reference crop evapotranspiration from temperature. Appl. Eng. Agric, 1(2), 96-99.

Heredia, J, and Meléndez, M. 2016. Premodelo de parámetros agregados Precipitación-Escorrentía de las subcuencas media y alta del río Pas (Cantabría, España). Congreso Hispano Luso sobre las Aguas Subterráneas en el Segundo Ciclo de Planificación Hidrológica. AlHGrupo Español-AlH-Grupo Portugués. Madrid. Noviembre 2016.

Hernández-Quesada, P. 2015. Funcionamiento hidrogeológico del área de los barrancos de Moya y Azuaje, Norte de Gran Canaria. Universidad de Las Palmas de Gran Canaria. Tesis Doctoral, 220 pp.

ITGE. 1993. Estudio isotópico de las aguas subterráneas de la isla de La Palma. Madrid: Ministerio de Industria y Energía.

König, B. 1997. Determination of solute fluxes and balances in the groundwater of th volcanic island of La Palma (Canary Islands). Christian-Albrechts-Universität (Kiel, Germany): Berichte - Reports, Geol.-Paläont. Inst. Univ. Kiel. Tesis Doctoral, 201 pp. 
Lyell, C. 1865. Elements of Geology. Londres: 814 p. Obtenido de https://archive.org/details/in.ernet.dli. 2015.217064

Marrero, R. 2010. Modelo Hidrogeoquímico del acuífero de Las Cañadas del Teide, Tenerife, Islas Canarias. Universidad Politécnica de Cataluña. Barcelona. Tesis Doctoral, 501 pp.

Navarro, J. 1992. Capítulo III. Plan Hidrológico Insular. Excelentísimo Cabildo Insular de La Palma.

NRCS. 2004. National Engineering Handbook. Part 630: Hydrology, Chapter 10. National Resources Conservation Service. Obtenido de https://www.wcc.nrcs.usda.gov/ftpref/wntsc/H\&H/NEHh ydrology/ch10.pdf

Poncela, R. 2015. Hidrogeología del Sistema acuífero volcánico de La Palma (Islas Canarias). Alicante: Tesis Doctoral, $227 \mathrm{pp}$.

Poncela, R. and Skupien, E. 2013. Estado de las Masas de Agua Subterráneas de La Palma. Informe Técnico. Consejo Insular de Aguas de La Palma.

Skupien, E., and Poncela, R. 2011. Características hidrogeo- lógicas, químicas e isotópicas del agua subterránea del acuífero volcánico de las vertientes en el entorno de los nacientes Marcos y Cordero (La Palma, Islas Canarias). In: Cabrera, M. y Custodio, E. (ed.), El conocimiento de los recursos hídricos en Canarias cuatro décadas después del proyecto SPA-15. ISBN: 978-84-938046-0-2. Las Palmas de Gran Canaria, 87-92.

SPA-15. 1975. Estudio científico de los recursos de agua en las Islas Canarias (SPA/69/515). (Vol. 3 vol. + mapas). Madrid: Ministerio de Obras Públicas. Dirección General de Obras Hidráulicas. UNESCO-PNUD.

Thornthwaite, C. W. 1948. An approach toward a rational classification of climate. Geographical review, 38(1), 5594.

Veeger, A. 1991. Geochemical methods for evaluating the origin and evolution of ground water in volcanic rocks. University of Arizona:Tesis Doctoral. $241 \mathrm{pp}$.

Viña, A. 2004. Historia. La Caldera de Taburiente y su evolución histórica. In García Canseco, V. (ed.), Parque Nacional de la Caldera de Taburiente (págs. 194-208). Canseco Editores.

Recibido: julio 2018

Revisado: septiembre 2018

Aceptado: febrero 2019

Publicado: diciembre 2019 
